\title{
QUALITATIVE PROPERTIES OF THE PERIPHERAL SPECTRUM
}

\author{
JAROSLAV ZEMÁNEK \\ Institute of Mathematics, Polish Academy of Sciences \\ P.O. Box 21, 00-956 Warszawa, Poland \\ E-mail: zemanek@impan.gov.pl
}

Let $A(z)$ be an analytic operator-valued function, say, on the open unit disc $\mathbb{D}$. Suppose that the spectral radius of $A(z)$ is a constant $c \geq 0$ independent of $z \in \mathbb{D}$. Then also the peripheral spectrum

$$
S=\{\lambda \in \operatorname{Sp} A(z):|\lambda|=c\}
$$

is independent of $z \in \mathbb{D}$, see [1, Proposition 2].

Suppose that a point $\lambda \in S$ has some qualitative property with respect to the spectrum $\operatorname{Sp} A\left(z_{0}\right)$ for some $z_{0} \in \mathbb{D}$, e.g., it is an isolated point, like an essential singularity or a pole of the resolvent of the operator $A\left(z_{0}\right)$. Does it possess the same property for all $A(z), z \in \mathbb{D}$ ?

\section{References}

[1] E. Vesentini, Maximum theorems for spectra, in: Essays on Topology and Related Topics (Mémoires dédiés à Georges de Rham), Springer, Berlin, 1970, 111-117. 